\title{
Ultra-Short-Term Multistep Prediction of Wind Power Based on Representative Unit Method
}

\author{
Mao Yang (D), Lei Liu, Yang Cui, and Xin Su \\ School of Electrical Engineering, Northeast Electric Power University, Jilin 132012, China \\ Correspondence should be addressed to Mao Yang; yangmao820@163.com
}

Received 11 May 2018; Revised 28 August 2018; Accepted 18 September 2018; Published 28 October 2018

Academic Editor: Leonardo Acho

Copyright (c) 2018 Mao Yang et al. This is an open access article distributed under the Creative Commons Attribution License, which permits unrestricted use, distribution, and reproduction in any medium, provided the original work is properly cited.

\begin{abstract}
With the continuous expansion of wind power grid scale, wind power prediction is an important means to reduce the adverse impact of large-scale grid integration on power grid: the higher prediction accuracy, the better safety, and economy of grid operation. The existing research shows that the quality of input sample data directly affects the accuracy of wind power prediction. By the analysis of measured power data in wind farms, this paper proposes an ultra-short-term multistep prediction model of wind power based on representative unit method, which can fully excavate data information and select reasonable data samples. It uses the similarity measure of time series in data mining, spectral clustering, and correlation coefficient to select the representative units. The least squares support vector machine (LSSVM) model is used as a prediction model for outputs of the representative units. The power of the whole wind farm is obtained by statistical upscaling method. And the number of representative units has a certain impact on prediction accuracy. The case study shows that this method can effectively improve the prediction accuracy, and it can be used as pretreatment method of data. It has a wide range of adaptability.
\end{abstract}

\section{Introduction}

With the gradual prominence of energy and environmental issues, wind energy as the most promising new energy source for commercial exploitation and utilization has been paid more and more attention by all countries [1-4]. Compared to conventional energy sources, wind power has characteristics of fluctuation and intermittence. The characteristics of itself make capacity reliability low and cause difficulties of the balance of reactive power and reactive power for power grid. So wind power integration has a certain adverse effect on the stability of the voltage and frequency of the power system. The traditional power generation plan is based on the reliability of the power supply and the predictability of the load. The formulation and implementation of the power generation plan have a reliable guarantee. But the prediction level of wind farm output can not reach the degree of practical engineering. The formulation of power generation plan becomes difficult and the reliability is not guaranteed. In order to solve the problem of large-scale wind power integration, it is necessary to make accurate prediction of wind power. In this way, it can not only help the power system to make the corresponding scheduling control strategy in advance but also ensure the safe and stable operation of the power system.

Wind power prediction in accordance with the time scale is generally divided into ultra-short-term, short-term, and long-term predicting [5-7]. Ultra-short-term predicting generally refers to the prediction within $4 \mathrm{~h}[8]$. The predicting result is used for online optimal operation of the power system. Wind power predicting methods usually include time series analysis method such as the autoregressive moving average (ARMA) model [9] and artificial intelligence method based on historical wind power data such as artificial neural network (ANN) [10] and support vector machine (SVM) [11]. Recently, a modified SVM, known as least squaresSVM (LSSVM), was successfully employed in wind power prediction [12]. LSSVM demonstrates better performance than SVM and ANN in terms of computational burden, simplicity, and the probability of convergence to global minima [10].

The research object of this paper is the ultra-short-term wind power prediction. The ultra-short-term wind power 
prediction mainly depends on the historical data sample. By improving the performance of units and studying identification and complement methods of data, more relatively complete historical data of wind power can be obtained. After a large amount of wind power data is obtained, it is worth studying problem of how to pretreat the wind power data effectively. Through effective pretreatment of wind power data, the quality of data, the training time and speed of prediction model, and the prediction accuracy of wind power are finally improved. In recent years, domestic and foreign scholars have done a great deal of research on wind power prediction and have made a series of achievements [1316]. Some studies have shown that the good or bad of the processing of sample data directly affects the accuracy of wind power prediction. The historical data are processed reasonably, and the processed data samples are used as input of prediction models. This purpose is to make the input sample representative and effectively improve the accuracy of the prediction model. When using historical data predicted wind power, the common method is cluster. The correlation between different wind farms or different fan output sequences is an important basis for clustering historical data samples. Reference [17] used K-means to cluster the wind power under low wind speed and predicted wind power combined the neural network (NN). The computational results reported that the proposed model customization approach produced accurate prediction models using a small number of input parameters. Reference [18] presented that a method can cluster historical data by K-means. Selecting proper clusters' centroids and optimal groups of data to be used as input to the neural networks, the precision of the output is greatly increased. Reference [19] proposed an improved evaluation method of wind power curtailment based on EOF and hierarchical clustering method. The clustering result is used for selecting sample wind turbines and determining allocation coefficients. Reference [20] proposed a data mining approach, which consists of the K-means clustering method and bagging NN. It can not only increase the predicting accuracy but also reduce the computational complexity. Reference [21] built a model to address different weather regimes based on the K-means clustering of meteorological variables, including surface pressure, pressure gradient, atmospheric temperature, and wind direction. Specific power prediction systems are more reasonable which are built according to each subset of data. The above methods all use K-means and hierarchical clustering method to preprocess the historical sample data. Combined with the corresponding prediction method, it can reduce the input parameters of the prediction model and improve the accuracy of the output results. However, the number of historical samples required for $\mathrm{K}$ means clustering is large. The $\mathrm{K}$-means clustering requires higher sample space and the convergence rate is slow. When the sample space is not convex, there will be a case of falling into the local optimal solution, which has a great limitation. Spectral clustering can be a good solution to these situations.

This paper proposes a method of ultra-short-term multistep prediction of wind power based on representative unit method. Firstly, the similarity judgment method of time series in data mining is used to establish the similarity judgment matrix, and it is considered as input of spectrum clustering in order to obtain the clustering groups of units. Secondly, using the correlation coefficient selects representative units in each clustering groups. Quantum Particle Swarm Optimization (QPSO) optimizes the needed parameters in the process of modeling. Finally, using LSSVM completes the ultra-short-term prediction on the representative units and it gets the prediction of the whole wind farm on statistical upscaling. In this way, the processing of effective historical data samples is completed. It reduces the size of the input sample and the accuracy of wind power prediction is improved.

\section{Theoretical Basis}

2.1. Similarity Measurement of Wind Power Sequences. Due to the randomness and intermittent of wind, the outputs of wind power are different in size. Because of the various topography, wake effect, and other factors, the size of the wind power outputs of each unit is also different [22]. Therefore, it is beneficial to establish the predicting model by reasonably processing the wind power sequences of each unit. In this paper, similarity measure method in data mining is used as data pretreatment method for spectral clustering [23]. It can segment the wind power output sequence of unit and determine the similarity and judge the similarities between the two units by European distance.

Two equal wind power sequences are expressed as $X_{1}=$ $\left\{x_{11}, x_{12}, \ldots, x_{1 n}\right\}$ and $X_{2}=\left\{x_{21}, x_{22}, \ldots, x_{2 n}\right\}$. Then their European distance is defined as $D=\sqrt{\sum_{i=1}^{n}\left(x_{1 i}-x_{2 i}\right)^{2}}$. If the two wind power sequences are similar in shape, they cannot be classified into the same clustering group. Because the Euclidean distance does not meet the clustering conditions, in order to solve the above problems, the method based on transformation and criterion proposed by Agrawl is used to obtain the similarity judgment matrix of wind power sequence [24]. It is a measure of similarity in data mining, which can solve the problems of offset and noise of wind power sequences. The two morphological similar wind power sequences are shown in Figure 1.

The basic idea of obtaining the similar decision matrix of the power sequence is as follows. Using the window width as $n$, the original power sequence is divided into $m$ segments slidingly. In this case, the original sequence is $x=$ $\left\{v_{1}, v_{2}, \ldots, v_{m}\right\}$. The change of the deviation and amplitude is obtained by using

$$
v_{i j}^{\prime}=\frac{2 v_{i j}-\left(v_{\max }+v_{\min }\right)}{v_{\max }-v_{\min }} .
$$

In the equation, $v_{i j}$ is the No. $j$ value of subsequence $v_{i}$, $v_{\max }$ is the maximum value of $v_{i}$, and $v_{\min }$ is the minimum value of $v_{i} ; v_{i j}^{\prime}$ is the changed value of the No. $j$ element in the subsequence $v_{i}$.

According to (1), the $x$ sequence is calculated to obtain $X$. The values of the sequence $X$ are concentrated within $[-1,1]$, which solves the problem that the Euclidean distance is too large to obtain great accuracy of similarity judgment. 


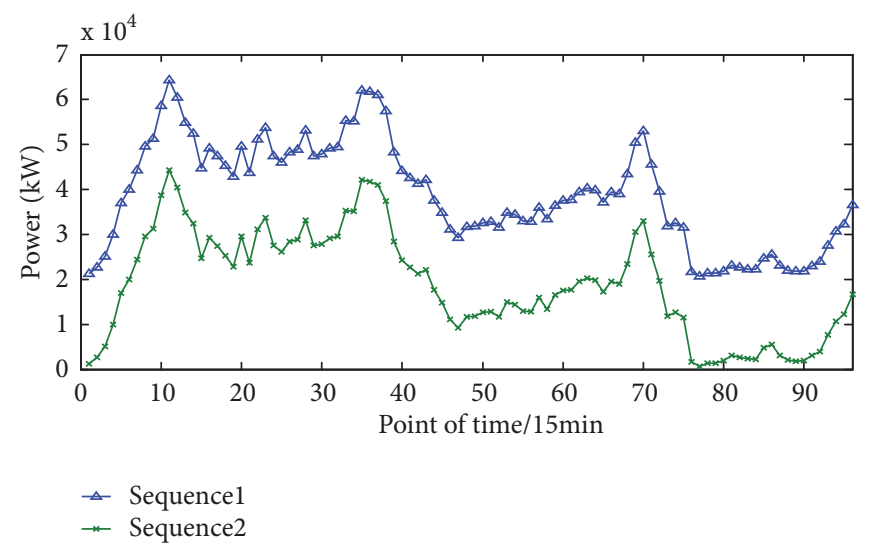

FIgURE 1: Schematic diagram of two morphologically similar sequences.

As shown in Figure 2, two morphological similar sequences after canonical transformation completely coincide.

The two transformed wind power sequences are $X_{1}=$ $\left\{v 1_{1}^{\prime}, v 1_{2}^{\prime}, \ldots, v 1_{m}^{\prime}\right\}$ and $X_{2}=\left\{v 2_{1}^{\prime}, v 2_{2}^{\prime}, \ldots, v 2_{m}^{\prime}\right\} . \varepsilon$ is the threshold of the Euclidean distance between $X_{1}$ and $X_{2}$. The number of European distances less than the threshold $\varepsilon$ will be recorded as $z$. If $X_{1}$ and $X_{2}$ satisfy the equation $\left|v 1_{i}^{\prime}-v 2_{i}^{\prime}\right|<$ $\varepsilon$ and the number $z$ is more than the setting value $\theta$, then they are similar and their degree of contact is 1 . Otherwise, their degree of contact is 0 . Therefore, a similarity matrix between $X_{1}$ and $X_{2}$ is established. The wind power sequences in the wind farm included $t$ units make similar judgments and generate the similarity judgment matrix $W$ which its dimension is $t \times t$ according to the above method.

In this paper, the window width is selected as 2 hours. In the same manner if the sampling interval is $15 \mathrm{~min}$, the window width is 8 . It uses QPSO algorithm to optimize the above threshold $\varepsilon$ and set value $\theta$. The fitness function of QPSO algorithm is the root mean square error as well as (8) [25].

2.2. Spectral Clustering Algorithm. In order to accurately obtain clustering groups based on the wind power output of units in the wind farm, it is planned to use SC algorithm for clustering [26]. The idea of SC comes from spectral partition theory. Assuming each sample data is thought as the vertex $V$ in the graph and the edge $E$ between the vertexes assignments weight value $W$, it can get an undirected weighting graph $G=(\mathrm{V}, \mathrm{E})$. Therefore, the clustering problem is transformed into the graph division problem in the graph. SC has the clustering ability to recognize nonconvex distributed, which can cluster in any shape of sample space and converge to the global optimal solution. However, the key to obtaining the high accuracy result of SC is how to create a similarity matrix, which can effectively improve the similarity between similar points and reduce the similarity between different points. Therefore, this paper uses the similarity judgment matrix based on the criterion transformation as the similarity matrix of the spectral clustering, which makes cluster more reasonable and improves the clustering quality.
In recent years, it has been found that multipath segmentation clustering is better than 2-path segmentation clustering. This paper uses the Ng-Jordan-Weiss (NJW) of spectral clustering algorithm [27]. The main process is described as follows.

Suppose the sample dataset is $S=\left\{s_{1}, s_{2}, \ldots, s_{n}\right\}$.

Step 1. $W$ is similarity matrix of $S$, which is obtained according to canonical transformation of (1).

Step 2. The Lagrange matrix is L. Degree matrix is $D$. They are satisfied $L=D-W . D$ can be expressed as follows:

$$
D_{i j}= \begin{cases}\sum_{j=1, j \neq i}^{n} w_{i j}, & i=j \\ 0, & i \neq j,\end{cases}
$$

Step 3. $1 \geq \lambda_{1} \geq \lambda_{2} \cdots \geq \lambda_{n}$ is obtained by calculating the eigenvalue of $L$ and arranging in descending order. Preceding $k$ eigenvalues construct the matrix as $\lambda=$ $\left[1, \lambda_{1}, \lambda_{2}, \ldots, \lambda_{k-1}\right] \in R^{\mathrm{n} \times k}$.

Step 4. The row vector of matrix $\lambda$ is transformed into a unit vector, resulting in a matrix $Y$ as shown in the following:

$$
Y_{\mathrm{ij}}=\frac{\lambda_{i j}}{\sqrt{\sum_{j=1}^{n} \lambda_{i j}^{2}}} .
$$

Step 5. Each row of matrix $Y$ can be considered as a point in space $R^{\mathrm{k}}$, and using K-means algorithm or any other classical algorithm gets $k$ clusters.

Step 6. The data points $y_{i}$ are divided into clusters $j$, only if the No. $i$ row of $Y$ is divided into clusters $j$.

When the No. $k+1$ eigenvalue compared to the No. $k$ eigenvalue changes greatly, the feature matrix formed by the first $k$ eigenvectors, which represents the stable relationship between elements. The preceding $k$ eigenvalues are called dominant eigenvalues, and the corresponding eigenvectors 


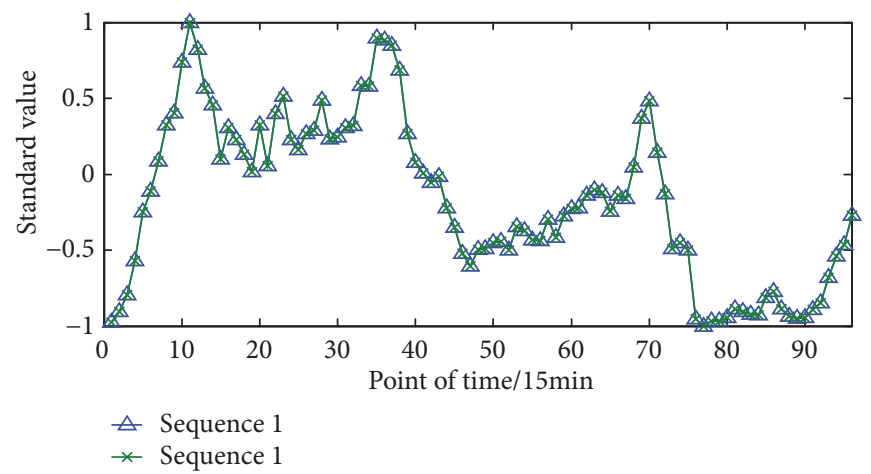

FIGURE 2: Schematic diagram of two sequences after canonical transformation.

are called dominant eigenvectors. Therefore, it is determined that the number of clusters is $k$.

The data comes from a wind farm in northeastern of China. The installed capacity of a single unit is $1500 \mathrm{~kW}$ and there is a total of 33 units. The total installed capacity is $49500 \mathrm{~kW}$. The height of wind turbine hub is $70 \mathrm{~m}$. The rated wind speed is $11 \mathrm{~m} / \mathrm{s}$. The working speed is from $3 \mathrm{~m} / \mathrm{s}$ to $25 \mathrm{~m} / \mathrm{s}$. The sampling interval is $15 \mathrm{~min}$. There are 96 data points in a day. By SC of wind power data, the number of dominant eigenvalues is 3 . So the number of clustering groups is $k=3$. Figure 3 shows an example of the wind power output sequence of three units in the same clustering group. Figure 4 shows an example of the wind power output sequence of one unit of the three clustering groups. Figure 3 shows the same clustering group of wind power output is similar in size and Figure 4 shows the different clustering groups of wind power output are different in size. It shows that SC is effective. Wind power outputs with similar fluctuate characteristics are divided into one clustering group. Wind power outputs with different fluctuate characteristics are divided into different clustering groups.

2.3. Select Representative Unit. After clustering groups are selected, one or several representative units are selected in each clustering group, usually one. The representative unit is measured according to the similarity between the wind power outputs of the representative unit and the total wind power outputs of the corresponding clustering group. It tries to choose the unit with high similarity as much as possible. The representative unit is selected according to the correlation coefficient $R$ as (4) shows

$$
R=\frac{\sum_{i=1}^{n}\left(p_{t, i}-\overline{p_{t}}\right)\left(p_{k, i}-\overline{p_{k}}\right)}{\sqrt{\sum_{i=1}^{n}\left(p_{t, i}-\overline{p_{t}}\right)^{2}} \sqrt{\sum_{i=1}^{n}\left(p_{k, i}-\overline{p_{k}}\right)^{2}}}
$$

In (4), $p_{t, i}$ is the wind power output sequence of the No. $t$ unit in the No. $k$ clustering group; $\overline{p_{t}}$ is the average value of wind power output sequence of the No. $t$ unit in the No. $k$ clustering group; $p_{k, i}$ is the wind power output sequence of the No. $k$ clustering group; $\overline{p_{k}}$ the average value of wind power output sequence of the No. $k$ clustering group.

After the representative unit finishes selection, the wind power outputs of this clustering group can be predicted according to the weight coefficient. The weight coefficient $b_{k}$ is the ratio of the total installed capacity of all the units in this cluster group and the installed capacity of the representative unit. The representative unit belong to the same cluster group. According to the following equation, the wind power outputs of the whole wind farm can be predicted.

$$
p_{a l l, j}=\sum b_{k} p_{t, j}
$$

$P_{a l l, j}$ is the prediction of wind power outputs of the whole wind farm; $p_{t, j}$ is the prediction of wind power outputs of the representative unit $t ; b_{k}$ is the weight coefficient of the No. $k$ clustering group. The predictions of wind power outputs of all clustering groups summed up the prediction of wind power outputs of the whole wind farm on statistical upscaling method.

This section mainly expounds the necessary algorithm and process for selecting units. One or more representative units in the cluster category are selected to replace all the unit data. It can improve the prediction accuracy and reduce the computational cost. In the introduction, it has been shown that clustering the wind power history output sequence can improve the prediction accuracy. Therefore, it uses the spectral clustering which is better than the traditional K-means algorithm to cluster the wind power history output sequence. As the input of spectral clustering, similarity judgment matrix has great influence on spectral clustering. Therefore, it is necessary to process the wind power historical output sequence and obtain the similarity judgment matrix. Based on the standard transformation method in data mining, the wind power historical output sequence of wind power is processed. It solves the problems of wind power sequence migration, noise and so on. To enhance the correlation degree between units with high similarity, weaken the correlation degree between fans with low similarity. Finally, the similarity judgment matrix is generated to guarantee the validity and reliability of spectral clustering. After clustering, through the calculation of correlation coefficient, it can get the wind unit number and the corresponding wind power output sequence The wind power historical output sequence of the unit is used as the input of the prediction model. The predicted result is multiplied by the weight coefficient. The final value of the whole wind farm is finally obtained by statistical upscaling method. 


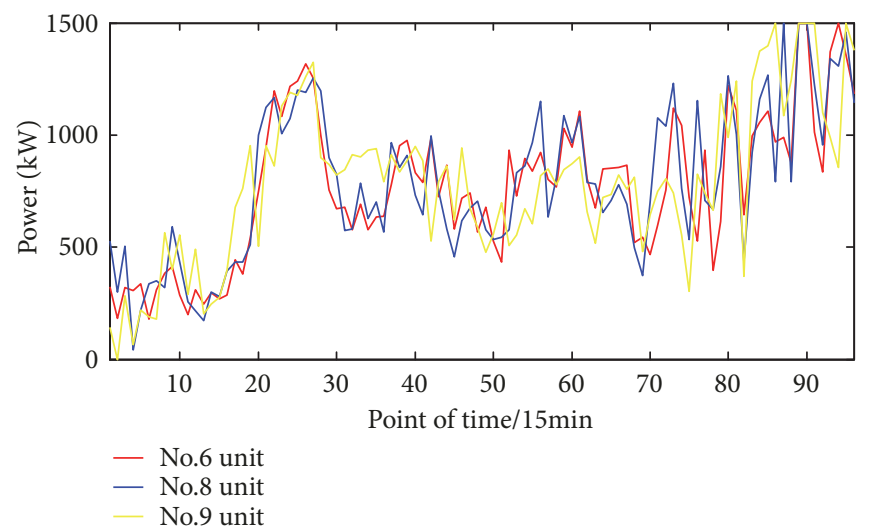

Figure 3: An example of the wind power output sequence of three units in the same clustering group.

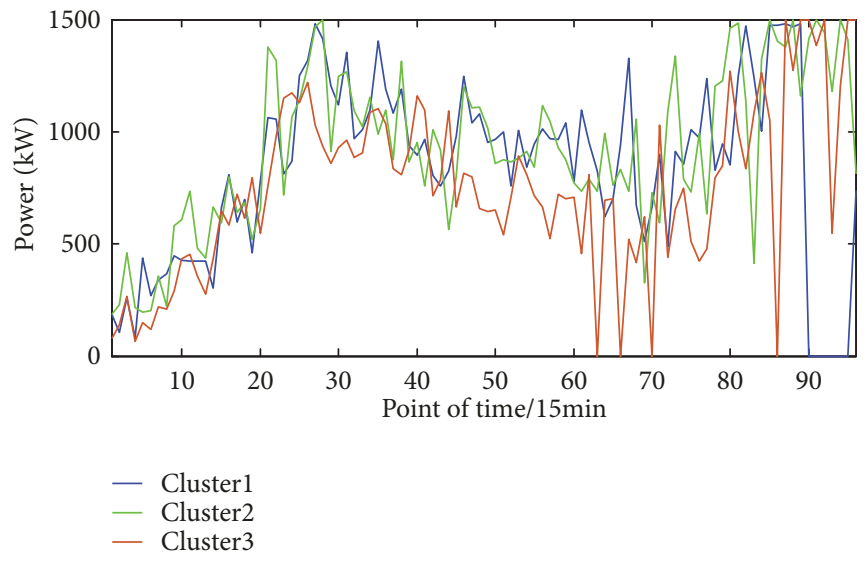

Figure 4: An example of the wind power output sequence of one unit of the three clustering groups.

\section{Prediction Model of Wind Power Based on R-SC-LSSVM}

3.1. R-SC-LSSVM Model. This paper uses the similarity measure method of time series in data mining, which can take full consideration of some problems, such as power sequence migration and noise. Then the QPSO is used to optimize parameters in similarity measurement process in order to get the reasonable similarity judgment matrix. The similarity judgment matrix is considered as input of SC in order to obtain the clustering groups of units. And by the correlation coefficient $\mathrm{R}$, the representative units in each clustering groups are selected. Using LSSVM completes the ultra-short-term prediction on the representative units and then it gets the prediction of the whole wind farm on statistical upscaling. Establish R-SC-LSSVM model to predict the wind power. Execution time for the whole multistep wind power prediction is less than $3 \mathrm{~s}$. The R-SC-LSSVM model predictive modeling process is shown in Figure 5.

3.2. The Evaluation Function of Prediction Error. Select the accuracy rate, pass rate, root mean square error, and mean absolute percentage error of the four indicators to assess the prediction effect. The four indicators are as follows.
(1) Accuracy rate $r_{1}$ is described as follows:

$$
r_{1}=\left[1-\sqrt{\frac{1}{16} \sum_{k=1}^{16}\left(\frac{P_{M}^{k}-P_{p}^{k}}{\operatorname{Cap}}\right)^{2}}\right] \times 100 \%,
$$

(2) Pass rate $r_{2}$ is described as follows:

$$
r_{2}=\frac{1}{16} \sum_{k=1}^{16} B^{k} \times 100 \%
$$

If $\left(1-\left|P_{M}^{k}-P_{P}^{k}\right| / C a p\right) \times 100 \% \geq 85 \%, B^{k}=1$, or $B^{k}=0 . B^{k}=1$ indicates that the prediction is in line with the standard; however $B^{k}=0$ indicates that the prediction is out of line with the standard.

(3) Root mean square error $r_{3}$ is described as follows.

$$
r_{3}=\sqrt{\frac{1}{16} \sum_{k=1}^{16}\left(\frac{P_{M}^{k}-P_{P}^{k}}{\operatorname{Cap}}\right)^{2}} \times 100 \%,
$$




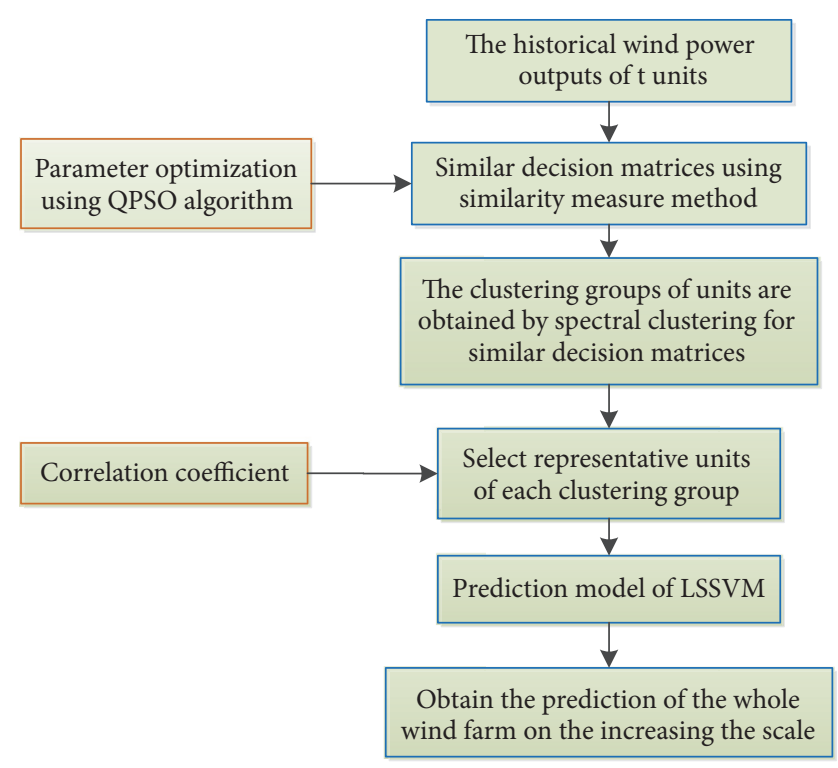

FIGURE 5: Model of R-SC-LSSVM.

(4) Mean absolute percentage error $r_{4}$ is described as follows.

$$
r_{4}=\frac{1}{16} \sum_{k=1}^{16}\left|\frac{P_{M}^{k}-P_{P}^{k}}{P_{M}^{k}}\right| \times 100 \%,
$$

The specific meanings of variables above three indicators are as follows.

In the equations, $P_{M}^{k}$ is the actual power at No. $k . P_{P}^{k}$ is the prediction power at No. $k$. The wind farm installed capacity is Cap.

\section{Case Studies}

4.1. Description Data. Taking the measured wind power data of a wind farm in northeast of China is used as the experimental sample. The installed capacity of a single unit is $1500 \mathrm{~kW}$. The total capacity of the wind farm is $49500 \mathrm{~kW}$, which has 33 units. This paper selects 2016 time points about 21 days' wind power data, and its sampling intervals are 15 minutes. The data of the previous day is used as a training set. It predicts the wind power in the next 4 hours or 16 points. The actual wind power is shown in Figure 6.

4.2. R-SC-LSSVM Model Prediction. Using the data of the previous day as the training data, the data of the previous day is processed for clustering. The previous day's power sequence matrix $x=\left\{x_{1}^{\mathrm{T}}, x^{\mathrm{T}}, \ldots, x_{33}^{\mathrm{T}}\right\}$ can be transformed into $X=$ $\left\{X_{1}^{\mathrm{T}}, X_{2}^{\mathrm{T}}, \ldots, X_{33}^{\mathrm{T}}\right\}$ according to (1), among $x_{i}^{\mathrm{T}}$ is the power sequence of the No. $i$ unit for one day. Then the data in the $X$ has similarity determination for getting a similar judgment matrix $W$. If the No. $j$ and the No. $j$ units have similar power sequences, the value in the matrix is $W(i, j)=1$.

Take the 9th day at 12:00 as an example, using the QPSO algorithm optimizes the two parameters the threshold $\varepsilon$ and the setting value $\theta$, which are needed for obtaining the similar judgment matrix. When the population size is 20 , the maximum number of iterations is 200 and the shrinkage expansion factor $\alpha=0.8$; we can get that the optimization threshold is $\varepsilon=2.3251$ and the optimization setting value is $\theta=40$. Then the similar judgment matrix is obtained. The similarity judgment matrix is used as the input sample data for SC. The SC can determine the number of clusters by calculating the eigenvalues of the Lagrange $L$. The eigenvalues are shown in (10). The value eigenvalues of unit are found in Figure 7.

$$
\begin{aligned}
X & =\left[x_{1}, x_{2}, x_{3}, x_{4}, \ldots, x_{33}\right] \\
& =[1.00,0.94,0.85,0.53,0.53, \ldots, 0.18] .
\end{aligned}
$$

According to the observation, the eigenvalues $x_{1}, x_{2}, x_{3}$ are gradually decreasing but $x_{4}$ is suddenly decreasing, and the change amplitude of the eigenvalue after $x_{4}$ is obviously reduced. According to Section 2.2, the units can be divided into three categories. Then the clustering units with similar output can be obtained. The output curves of the whole wind farm and the three types of cluster units are shown in Figure 8, and the results of cluster are shown in Table 1.

The closer the unit's number is, the smaller the distance between the units is and the higher the similarity of the units' output. But due to the randomness of the wind, the difference in the topography and geomorphology of each unit, the wake effect of units, and so on, the number of individual units in the clusters is quite different. It can be seen that the clustering result is better, and the output of clustering units from more to less in turn is from clustering groups 1 to 3 . After the units' clustering is completed, one representative unit is selected in each category. Selecting the representative unit according to the correlation coefficient, the result is shown in Table 2. The output curve of the representative unit is shown in Figure 9.

Comparing Figure 9, we can know that the fluctuations of unit No.32 is similar to unit 28 in a period of $0-20$ time points, but it is quite different from unit No.19. In a period of 20-40 time points, the fluctuations of unit No.19 are similar to unit No.28, but they are quite different from unit No.32. In a period of 40-70 time points, the fluctuations of units No.19, No.28, and No.32 have smaller similarity. In a period of 70-96 time points, the output of units No.19, No.28, and No.32 is almost 0. It shows that the selected representative contains sufficient information; what is more it also verifies that selecting the representative unit is reasonable and the clustering is effective. After selecting the representative unit of each clustering groups, the proposed method can be compared with SC-LSSVM and LSSVM; when predicting the entire wind power, the main ideas of the two methods are as follows.

The idea of SC-LSSVM is using the wind power sequence of 33 units to generate the similarity judgment matrix by using the above method; then the similarity judgment matrix is clustered by SC to obtain the clustering groups. The LSSVM model is established for the total wind power of each clustering group, finally, adding the prediction results of each clustering as the whole power prediction value. The thought 


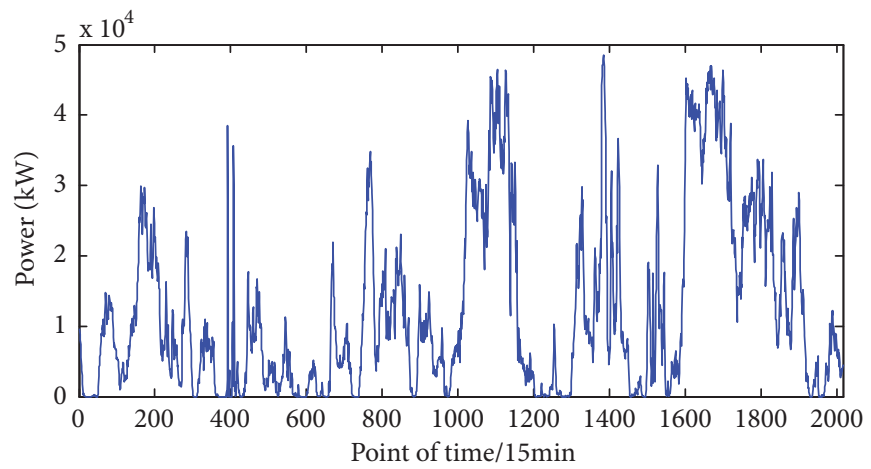

Figure 6: The actual wind power sequence.

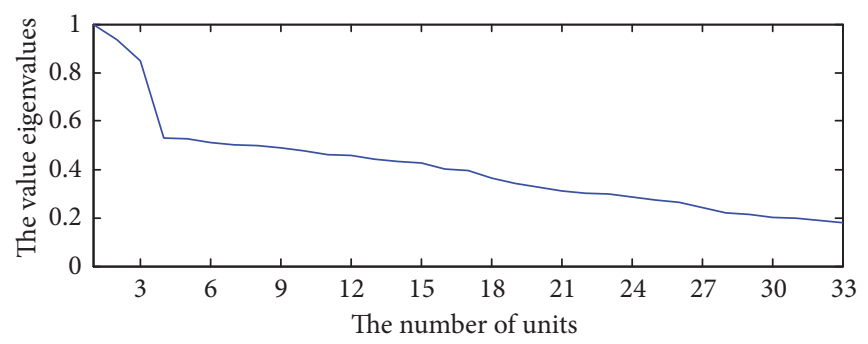

Figure 7: The value eigenvalues of units.

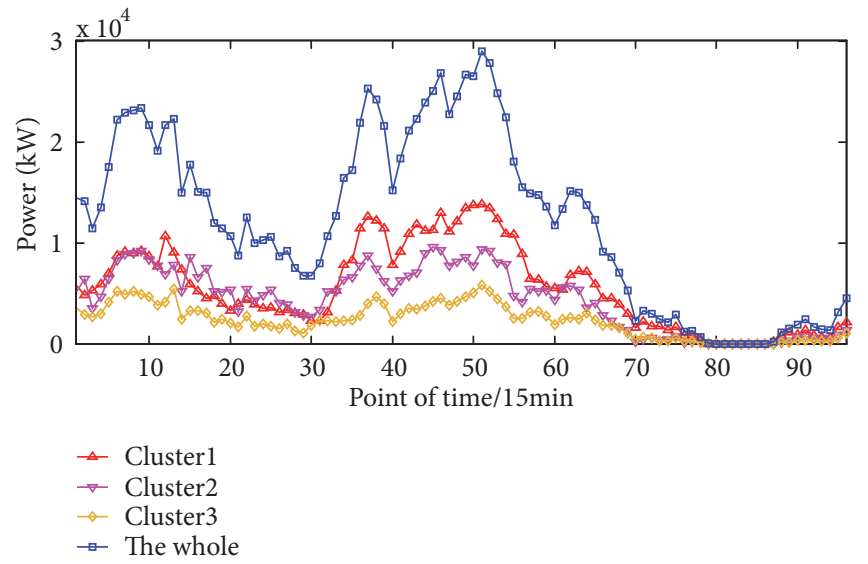

Figure 8: Comparison of the whole output curve and the output curve of three cluster machines.

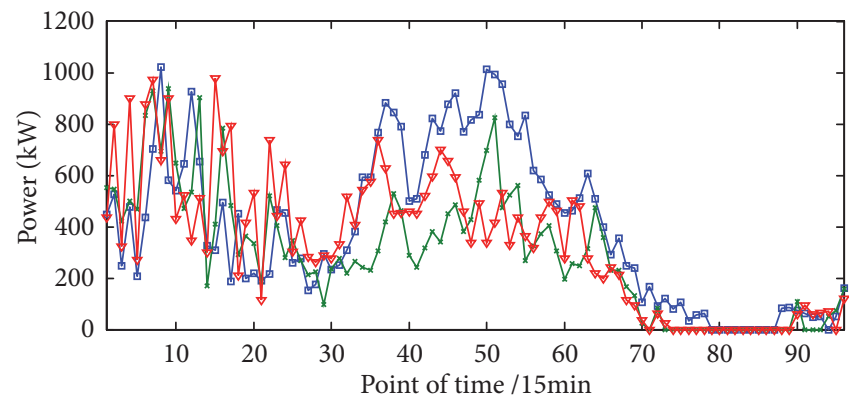

$\rightarrow$ No. 19 unit

* No. 32 unit

$\rightarrow$ No. 28 unit

FIGURE 9: Output curve of representative unit. 
TABLE 1: Clustering results of wind turbine groups.

\begin{tabular}{|c|c|c|c|}
\hline Object & & Parameters & \\
\hline Unit number & & $1 \sim 33$ & \\
\hline $\begin{array}{l}\text { The number of } \\
\text { dominant eigenvalues }\end{array}$ & & 3 & \\
\hline $\begin{array}{l}\text { The number of } \\
\text { clustering group }\end{array}$ & No.1 clustering group & No. 2 clustering group & No.3 clustering group \\
\hline $\begin{array}{l}\text { The number of the same } \\
\text { clustering group unit }\end{array}$ & $12,13,14,15,16,17,18,19,20,22,25,26,27$ & $2,3,4,5,6,7,8,9,21,23,31,32,33$ & $1,10,11,24,28,29,30$ \\
\hline
\end{tabular}

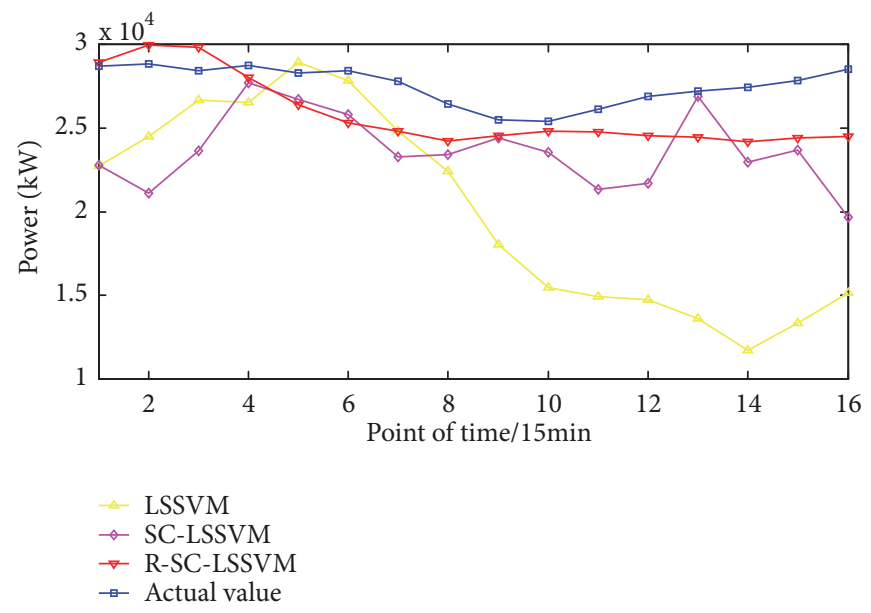

FIGURE 10: Comparison of predicted and actual values of three methods based on LSSVM.

TABLE 2: The number of representative unit.

\begin{tabular}{lc}
\hline The number of clustering group & $\begin{array}{c}\text { The number of representative } \\
\text { unit }\end{array}$ \\
\hline No.1 clustering group & 19 \\
No.2 clustering group & 32 \\
No.3 clustering group & 28 \\
\hline
\end{tabular}

of LSSVM is using the historical wind power directly to build a LSSVM model for predicting the entire wind power.

Figure 10 compares the three methods of wind power prediction and the actual power value; $r_{1}$ of this paper's model is $89.43 \%, r_{2}$ is $85.52 \% r_{3}$ is $11.07 \%$, and $r_{4}$ is $14.06 \%$. Compared to methods of SC-LSSVM and LSSVM, $r_{1}$ of this paper's model is improved to $2.99 \%$ and $4.69 \%, r_{2}$ of this paper's model is improved to $9.28 \%$ and $15.15 \%, r_{3}$ of this paper's model is improved to $2.51 \%$ and $4.20 \%$, and $r_{4}$ of this paper's model is improved to $3.48 \%$ and $5.37 \%$, respectively. It can be found that the prediction model of this paper is the best, comparing with the methods of SC-LSSVM and LSSVM. The results are shown in Table 3.

In order to illustrate the rationality and applicability of processing data with the method of the SC and representative unit, in addition to the combination of artificial intelligence algorithm in LSSVM, but also with time series method ARMA, we have the following is 3 methods: R-SC-ARMA prediction method is the 33 units of wind power generation
TABLE 3: Prediction results of three methods based on LSSVM.

\begin{tabular}{lccc}
\hline Indicators & R-SC-LSSVM & SC-LSSVM & LSSVM \\
\hline $\mathrm{r}_{1}(\%)$ & 89.43 & 86.44 & 84.74 \\
$\mathrm{r}_{2}(\%)$ & 85.52 & 76.24 & 70.37 \\
$\mathrm{r}_{3}(\%)$ & 11.07 & 13.58 & 15.27 \\
$\mathrm{r}_{4}(\%)$ & 14.06 & 17.54 & 19.43 \\
\hline
\end{tabular}

TABLE 4: Prediction results of three methods based on ARMA.

\begin{tabular}{lccc}
\hline Indicators & R-SC-ARMA & SC-ARMA & ARMA \\
\hline $\mathrm{r}_{1}(\%)$ & 86.23 & 84.49 & 81.37 \\
$\mathrm{r}_{2}(\%)$ & 74.81 & 55.72 & 51.64 \\
$\mathrm{r}_{3}(\%)$ & 13.78 & 15.88 & 19.05 \\
$\mathrm{r}_{4}(\%)$ & 17.89 & 20.06 & 24.46 \\
\hline
\end{tabular}

sequence using the above method to obtain similarity matrix for SC; the cluster groups is obtained, and the representative fan in each category is selected; then predict the power output with the ARMA model. Finally, the prediction results of the whole wind farm are obtained by raising the scale; SC-ARMA is to build an ARMA model for each clustering group, the wind power of each clustering group is obtained. Adding the prediction results of each clustering group as a whole power prediction, the ARMA is using the historical wind power directly to predict the wind power of the whole field by the ARMA model. The results are shown in Table 4. It can 


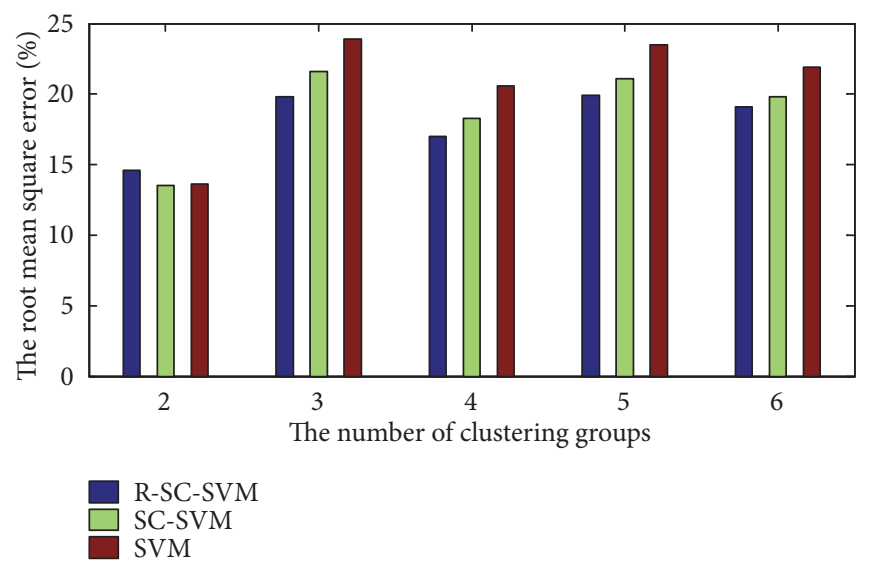

Figure 11: The root mean square error of three predicting methods.

TABLE 5: Comparison of prediction results based on LSSVM.

\begin{tabular}{|c|c|c|c|c|c|c|c|c|c|c|c|c|}
\hline \multirow{2}{*}{ Days } & \multicolumn{4}{|c|}{ R-SC-LSSVM } & \multicolumn{4}{|c|}{ SC-LSSVM } & \multicolumn{4}{|c|}{ LSSVM } \\
\hline & $\mathbf{r}_{1}(\%)$ & $\mathbf{r}_{2}(\%)$ & $\mathbf{r}_{3}(\%)$ & $\mathbf{r}_{4}(\%)$ & $r_{1}(\%)$ & $\mathbf{r}_{2}(\%)$ & $\mathbf{r}_{3}(\%)$ & $\mathbf{r}_{4}(\%)$ & $r_{1}(\%)$ & $\mathbf{r}_{2}(\%)$ & $\mathbf{r}_{3}(\%)$ & $\mathbf{r}_{4}(\%)$ \\
\hline 1 & 88.51 & 74.34 & 11.55 & 16.43 & 87.63 & 72.25 & 12.24 & 17.97 & 85.17 & 71.64 & 14.89 & 19.46 \\
\hline 5 & 88.03 & 73.57 & 12.09 & 16.56 & 87.04 & 70.34 & 13.08 & 18.45 & 85.21 & 70.57 & 15.43 & 19.44 \\
\hline 10 & 85.32 & 69.82 & 15.27 & 19.74 & 84.21 & 68.75 & 16.07 & 20.76 & 83.34 & 67.83 & 16.93 & 22.37 \\
\hline 15 & 85.95 & 68.34 & 15.04 & 19.59 & 84.61 & 68.56 & 16.01 & 20.11 & 83.04 & 67.24 & 17.30 & 23.71 \\
\hline 20 & 87.76 & 72.75 & 13.01 & 18.48 & 85.15 & 65.24 & 15.17 & 19.99 & 84.17 & 69.86 & 16.01 & 20.68 \\
\hline
\end{tabular}

know R-SC-ARMA's $r_{1}$ is $86.23 \%$; compared to methods of SC-ARMA and ARMA the accuracy is improved to $1.74 \%$ and $4.86 \%$, respectively. R-SC-ARMA's $r_{2}$ is $74.81 \%$; compared to methods of SC-ARMA and ARMA the accuracy is improved to $19.09 \%$ and $23.17 \%$, respectively. R-SC-ARMA's $r_{3}$ is $13.78 \%$; compared to methods of SC-ARMA and ARMA the accuracy is improved to $2.10 \%$ and $5.27 \%$, respectively. R-SC-ARMA's $r_{4}$ is $17.89 \%$; compared to methods of SC-ARMA and ARMA the accuracy is improved to $2.17 \%$ and $6.57 \%$, respectively. It can be found that the prediction accuracy of R-SC-ARMA is the highest comparing with the methods of SC-ARMA and ARMA. The proposed method SVM and ARMA model combined with selecting representative unit have higher accuracy than that SVM and ARMA model directly predicted with historical wind power, which illustrates that the methods with selecting representative can process the historical data reasonably, and the processed data as the input of the prediction model can effectively improve the accuracy of the prediction model.

In order to further verify the superiority of this paper's model, these three prediction methods are statistically calculated on a long time scale. Each day, 96 times of ultra-shortterm multistep prediction were carried out. The $1,5,10,15$, and 20 days' average value of $r_{1}, r_{2}, r_{3}, r_{4}$ are shown in Table 5 . From Table 5, it can be found that the R-SC-LSSVM is still superior to the other two methods on a long time scale. It shows that the sample data is analyzed and processed reasonably and then used as the input sample of the prediction model, which can effectively improve the prediction accuracy and has certain superiority and universal adaptability. The prediction result of SC-LSSVM is superior to LSSVM; it shows that the prediction accuracy can be improved by using the prediction model, which use the method of SC.

4.3. The Influence of Clustering Number on Prediction Results. The number of clustering group is the most important factor to predict the wind power. The SC can determine the number of clustering by calculating the eigenvalues of the Lagrangian matrix $L$. The number of clusters is different, when the wind power is clustered at different times. When we predict the whole field of wind power, $r_{3}$ of this paper's model and the other two methods at different cluster numbers is shown in Figure 11 .

The prediction error of R-SC-LSSVM is larger than that of LSSVM when the number of clustering group is 2, because the smoothing effect is not obvious when the number of representative units is less. Instead, the errors caused by the representative unit are amplified when calculating the entire wind power outputs. The prediction error of LSSVM is larger than that of SC-LSSVM, which is due to the high similarity of the wind power sequences in each category and the concentration of the wind power output. At the same time, when the number of clustering group is 2, the output power is often small and the root mean square error is smaller than that of other clusters. In this case, we can use SC-LSSVM instead of the proposed method in this paper to improve the prediction accuracy. 
When the number of clustering group is more than 2, the number of representative unit is larger and the smoothing effect is more obvious. The prediction results of R-SC-LSSVM are superior to those of SC-LSSVM and LSSVM.

\section{Conclusions}

In order to select the input samples rationally, this paper presents an ultra-short-term and multistep prediction model of wind power based on representative unit method, which can fully use the data mining information and improve the wind power prediction accuracy effectively.

The proposed method in this paper mainly consists of three steps: the establishment of similarity judgment matrix, obtaining the clustering group based on SC, and the selection of representative unit. These parts can be used to preprocess data, which makes the input data samples more reasonable and wider. It can be extended to other prediction models.

Compared with the method of SC-LSSVM and LSSVM, the prediction accuracy of wind power can be effectively improved when the representative unit of ultra-short-term wind power is selected based on R-SC-LSSVM. The number of clustering group is the most important factor for the prediction accuracy. When the number of clustering groups is 2 , the prediction accuracy of the proposed method in this paper is larger than the method of LSSVM. We can use SCLSSVM instead of the proposed method in this paper. When the number of clustering group is more than 2 , the prediction results of R-SC-LSSVM are superior to those of SC-LSSVM and LSSVM.

The number of selected representative units in this paper is 1 . The key point of next step is to discuss whether the number of selected representative unit and the distributions of the weight will influence the prediction results.

\section{Data Availability}

The data used to support the findings of this study are available from the corresponding author upon request.

\section{Conflicts of Interest}

The authors declare that they have no conflicts of interest.

\section{Acknowledgments}

The work described in this paper was supported by National Key R\&D Program of China (Technology and application of wind power / photovoltaic power prediction for promoting renewable energy consumption, 2018YFB0904200).

\section{References}

[1] S. Sun, F. Liu, S. Xue, M. Zeng, and F. Zeng, "Review on wind power development in China: Current situation and improvement strategies to realize future development," Renewable \& Sustainable Energy Reviews, vol. 45, pp. 589-599, 2015.
[2] Y. Feng, H. Lin, S. L. Ho et al., "Overview of wind power generation in China: Status and development," Renewable \& Sustainable Energy Reviews, vol. 50, pp. 847-858, 2015.

[3] Y. Zhang, N. Tang, Y. Niu, and X. Du, "Wind energy rejection in China: Current status, reasons and perspectives," Renewable \& Sustainable Energy Reviews, vol. 66, pp. 322-344, 2016.

[4] N. Safari, C. Y. Chung, and G. C. Price, "Novel multi-step short-term wind power prediction framework based on chaotic time series analysis and singular spectrum analysis," IEEE Transactions on Power Systems, vol. 33, no. 1, pp. 590-601, 2018.

[5] A. M. Foley, P. G. Leahy, A. Marvuglia, and E. J. McKeogh, "Current methods and advances in forecasting of wind power generation," Journal of Renewable Energy, vol. 37, no. 1, pp. 1-8, 2012.

[6] A. Tascikaraoglu and M. Uzunoglu, "A review of combined approaches for prediction of short-term wind speed and power," Renewable \& Sustainable Energy Reviews, vol. 34, pp. 243-254, 2014.

[7] P. Zhao, J. Wang, J. Xia, Y. Dai, Y. Sheng, and J. Yue, "Performance evaluation and accuracy enhancement of a day-ahead wind power forecasting system in China," Journal of Renewable Energy, vol. 43, pp. 234-241, 2012.

[8] L. Ma, S. Y. Luan, C. W. Jiang, H. L. Liu, and Y. Zhang, "A review on the forecasting of wind speed and generated power," Renewable \& Sustainable Energy Reviews, vol. 13, no. 4, pp. 915920, 2009.

[9] E. Erdem and J. Shi, "ARMA based approaches for forecasting the tuple of wind speed and direction," Applied Energy, vol. 88, no. 4, pp. 1405-1414, 2011.

[10] M. G. De Giorgi, S. Campilongo, A. Ficarella, and P. M. Congedo, "Comparison between wind power prediction models based on wavelet decomposition with least-squares support vector machine (LS-SVM) and artificial neural network (ANN)," Energies, vol. 7, no. 8, pp. 5251-5272, 2014.

[11] Q. Wu and C. Peng, "A least squares support vector machine optimized by cloud-based evolutionary algorithm for wind power generation prediction," Energies, vol. 9, no. 8, article 585, 2016.

[12] J. L. Wu, T. Y. Ji, M. S. Li, P. Z. Wu, and Q. H. Wu, "Multistep wind power forecast using mean trend detector and mathematical morphology-based local predictor," IEEE Transactions on Sustainable Energy, vol. 6, no. 4, pp. 1216-1223, 2015.

[13] L. Yang, M. He, J. Zhang, and V. Vittal, "Support-vectormachine-enhanced markov model for short-term wind power forecast," IEEE Transactions on Sustainable Energy, vol. 6, no. 3, pp. 791-799, 2015.

[14] G. Sideratos and N. D. Hatziargyriou, "An advanced statistical method for wind power forecasting," IEEE Transactions on Power Systems, vol. 22, no. 1, pp. 258-265, 2007.

[15] A. Bracale and P. De Falco, "An advanced bayesian method for short-term probabilistic forecasting of the generation of wind power," Energies, vol. 8, no. 9, pp. 10293-10314, 2015.

[16] Z. Shen and M. Ritter, "Forecasting volatility of wind power production," Applied Energy, vol. 176, pp. 295-308, 2016.

[17] A. Kusiak and W. Li, "Short-term prediction of wind power with a clustering approach," Journal of Renewable Energy, vol. 35, no. 10, pp. 2362-2369, 2010.

[18] M. Ghofrani, M. D. Rezende, R. Azimi, and M. Ghayekhloo, "Kmeans clustering with a new initialization approach for wind power forecasting," Transmission \& Distribution Conference \& Exposition, pp. 1-5, 2016. 
[19] Y. Wang, Z. Lu, Y. Qiao, Z. Cui, and R. Sun, "Wind power curtailment evaluation based on EOF and hierarchical clustering method," in Proceedings of the 2016 International Conference on Probabilistic Methods Applied to Power Systems (PMAPS), pp. 1-6, Beijing, China, October 2016.

[20] W. Wu and M. Peng, "A data mining approach combining Kmeans clustering with bagging neural network for short-term wind power forecasting," IEEE Internet of Things Journal, vol. 4, no. 4, pp. 979-986, 2017.

[21] Y. Li, C. Dai, T. Wang et al., "Separate wind power and ramp predictions based on meteorological variables and clustering method," in Proceedings of the 2016 IEEE 6th International Conference on Power Systems (ICPS), pp. 1-6, New Delhi, India, March 2016.

[22] J. Tian, D. Zhou, C. Su, M. Soltani, Z. Chen, and F. Blaabjerg, "Wind turbine power curve design for optimal power generation in wind farms considering wake effect," Energies, vol. 10, no. 3, pp. 395-414, 2017.

[23] M. Zhang and D. Pi, "A new time series representation model and corresponding similarity measure for fast and accurate similarity detection," IEEE Access, vol. 5, pp. 24503-24519, 2017.

[24] I. H. Witten, E. Frank, and M. A. Hall, Data Mining: Practical Machine Learning Tools and Techniques, Machinery Industry Press, 2005.

[25] L.-L. Peng, G.-F. Fan, M.-L. Huang, and W.-C. Hong, "Hybridizing DEMD and quantum PSO with SVR in electric load forecasting," Energies, vol. 9, no. 3, 2016.

[26] D. Liu, J. Wang, and H. Wang, "Short-term wind speed forecasting based on spectral clustering and optimised echo state networks," Journal of Renewable Energy, vol. 78, pp. 599-608, 2015.

[27] B. Huang and P. Yang, "A Modified Spectral Clustering Algorithm Based on NJW," in Proceedings of the EEE International Symposium on Knowledge Acquisition \& Modeling Workshop, pp. 381-384, Wuhan, China, December 2008. 


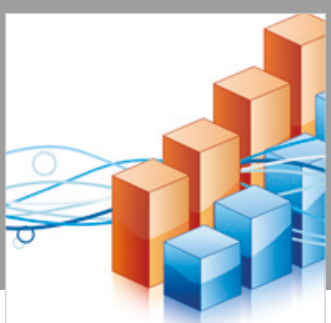

Advances in

Operations Research

\section{-n-m}
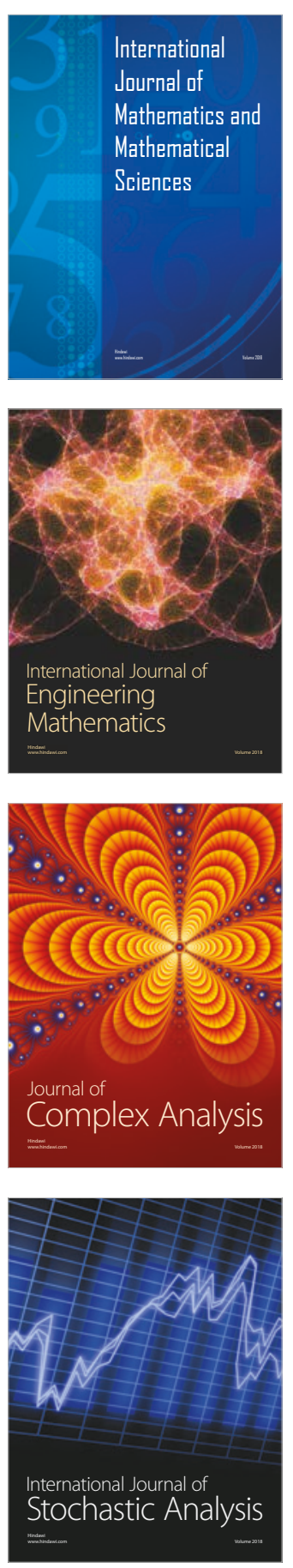
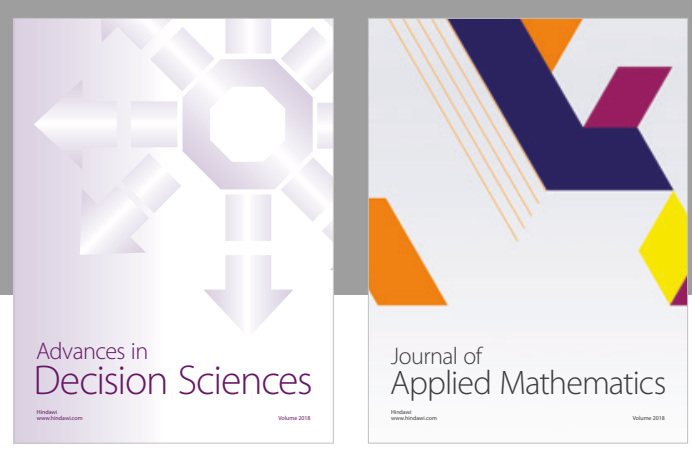

Journal of

Applied Mathematics
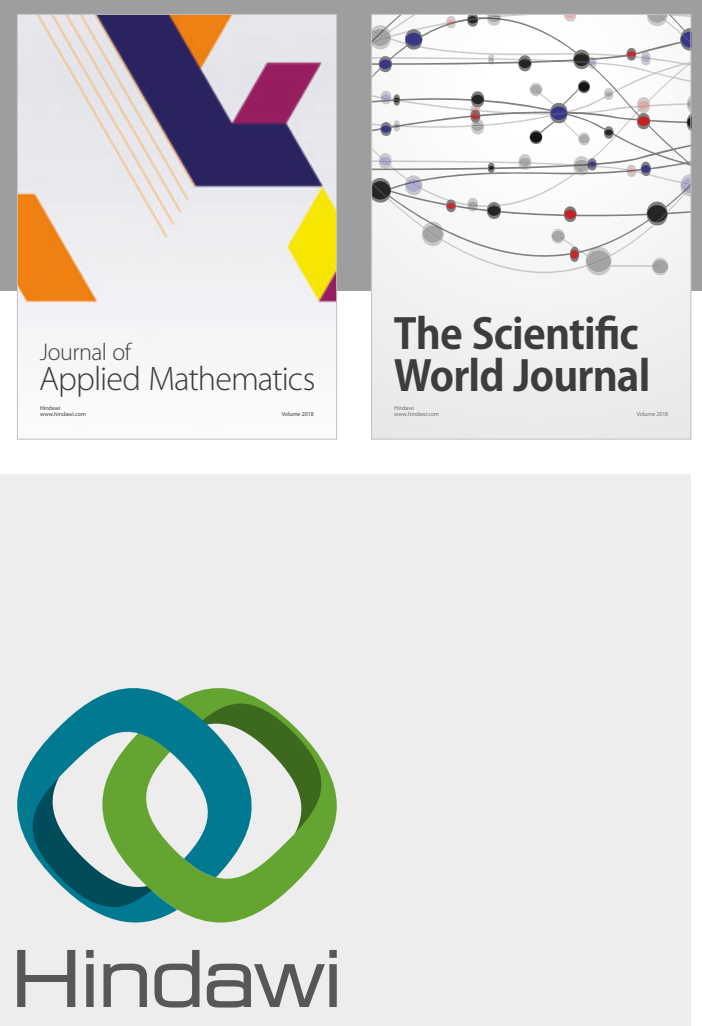

Submit your manuscripts at

www.hindawi.com

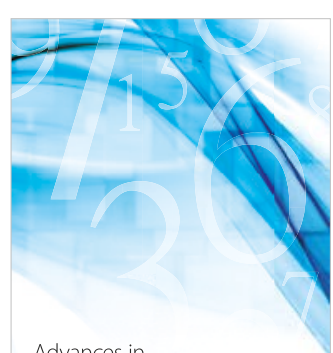

Advances in
Numerical Analysis
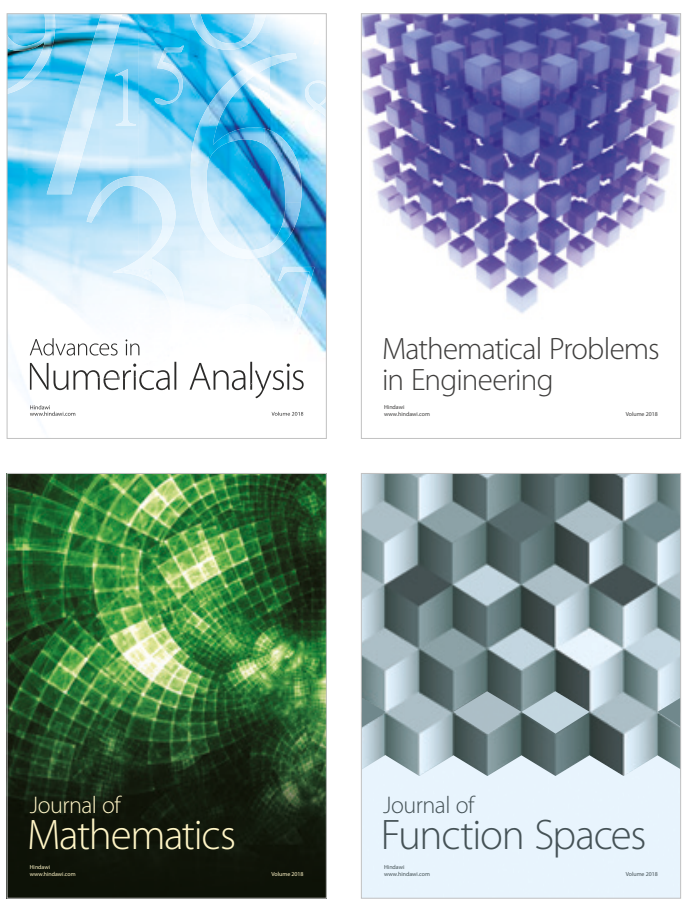

Mathematical Problems in Engineering

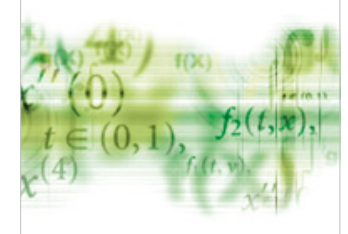

International Journal of

Differential Equations

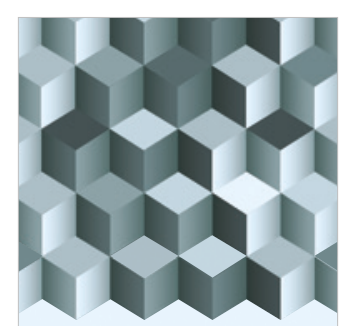

Journal of

Function Spaces

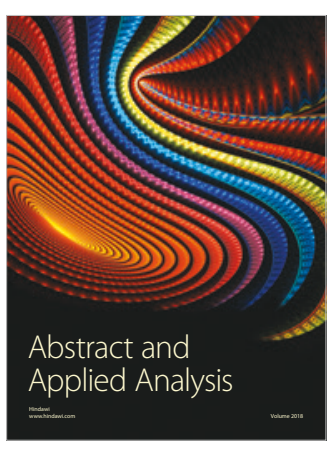

The Scientific

World Journal

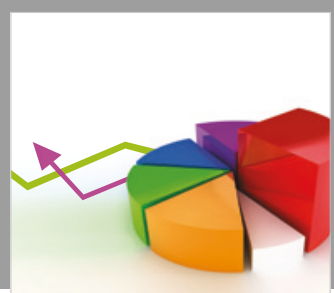

Journal of

Probability and Statistics
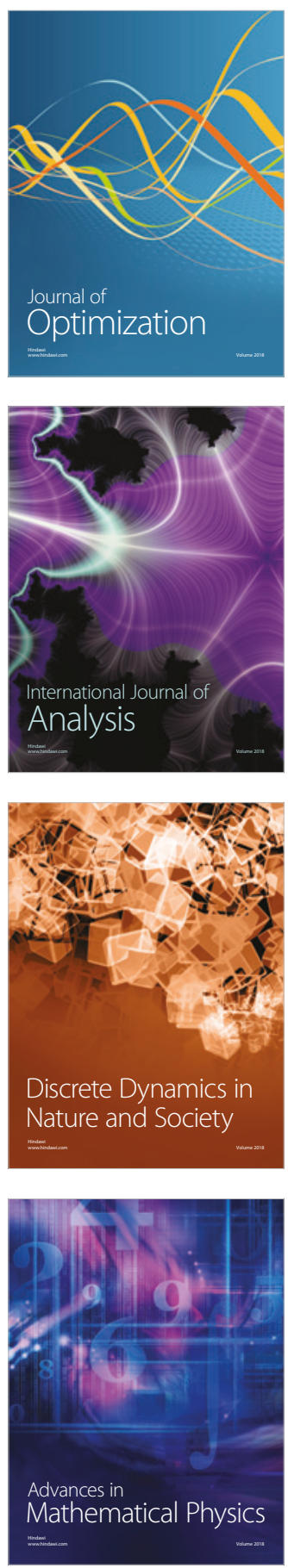